\title{
Aripiprazole for the Treatment of Inappropriate Sexual Behavior: Case Report of an Alzheimer's Disease Patient Known as Heterosexual with Recently Shifted Sexual Orientation to Same Gender
}

\author{
Sevda Sarikaya ${ }^{\mathrm{a}, *}$ and Basar Sarikaya ${ }^{\mathrm{b}}$ \\ a Private neurologist (dementia clinic), Istanbul, Turkey \\ ${ }^{\mathrm{b}}$ Department of Radiology, Neuroradiology, Yeditepe University Medical Faculty, Istanbul, Turkey
}

\begin{abstract}
Although inappropriate sexual behavior in dementia is not common, it can be extremely troublesome for healthcare professionals and caregivers as well as patients themselves. There is paucity of data in the literature for the management of these behaviors, but the limited available data suggest efficiency of some commonly used treatment modalities. Herein, we present a 74-year old Alzheimer's disease (AD) patient, known to be heterosexual throughout her past life, presenting with a recent display of same-sex inappropriate sexual behavior six years after the initial diagnosis of her disease. This new clinical picture of hypersexuality responded well to aripiprazole and her symptoms gradually diminished and eventually disappeared after onset of medication. This report also constitutes the first reported case of an AD patient in the published literature presenting with hypersexuality of shifted sexual orientation during the course of the disease.
\end{abstract}

Keywords: Alzheimer's disease, antipsychotic agents, aripiprazole, dementia, frontotemporal dementia, sexuality

\section{INTRODUCTION}

A broad spectrum of behavioral and psychological symptoms can develop in Alzheimer's disease (AD) and related dementias [1]. Inappropriate sexual behavior (ISB) is a potentially disruptive form of symptoms in people with dementia which is not very uncommon. It may cause considerable distress and potential risks [2]. ISB can include verbal or physical abnormal behavior. The physical manifestations may include sexual touching, fondling, disrobing, masturbation, and sexual intercourse attempts [3]. The occurrence of ISB in demented individuals

\footnotetext{
*Correspondence to: Sevda Sarikaya, Barbaros Mahallesi Ardic Sokak Varyap Meridian C blok Daire:70 Atasehir, Istanbul, Turkey. Tel.: +90 5327707917; E-mail: drsevdasarikaya@gmail. com.
}

reportedly ranges from 7 to $25 \%$, with higher prevalence in residents of skilled nursing facilities and in patients with more severe cognitive impairment [2]. Physical components appear to be more frequent in males [4], whereas women are likely to be more verbal [5].

In this report, we present a 74-year old AD patient who had been heterosexual in her past life, having exhibited same-sex ISB six years after the initial diagnosis of the disease successfully treated with aripiprazole. Her guardian fully read this manuscript and signed the informed consent form permitting to publish the information about the patient while respecting anonymity.

The fact that caregivers of the dementia patients are usually reluctant to disclose ISB due to social concerns, has led us to opine that ISB might be 
more prevalent than it is actually reported. Pubmed search was conducted by using the following search terms and strings: dementia; Alzheimer's; "sexual orientation"; "changed sexual orientation"; "sexual behavior"; and "inappropriate sexual behavior" that yielded no previous report of dementia related ISB with a change in sexual orientation throughout the course of the disease.

\section{CASE REPORT}

A 74-year-old female patient who had been followed at our private clinic for $\mathrm{AD}$ for the past two years, recently presented to her follow-up visit with the complaints of displaying hypersexual behavior to the females around her, including her female caregivers and adult granddaughters. According to the history obtained from her caregivers, this hypersexuality involved not only flirtatious remarks to females but also included attempts to touch their breasts and genitalia. The family was at first terrified and baffled by these new symptoms and preferred to ignore when they first became apparent in the past few months, but later on wanted to seek professional help as it was getting harder for them to find professional female caregivers to take her on; and also leading to social isolation of the family members. Eventually she was taken to a nursing home, where she continued to display ISB toward the female staff. During the clinical visits, she also made compliments to the female staff members and expressed a desire to be physically intimate with them.

Past history obtained from her son and daughter-in law revealed that she married with her first boyfriend when she was 17 years old and stayed married for 43 years until her husband passed away 14 years ago. Both of them were born and raised in Romania, but then immigrated to Turkey when she was at the age of 40. Reportedly, she had a decent and happy marriage and raised three children with her husband. She continued to work as a nurse in Turkey for 17 years until her retirement. After her spouse's death, she moved back and forth between Romania and Turkey spending half of the year at two homes in each country for a while but she was finally settled down permanently in Turkey in the past 8 years. She did not have another boyfriend after her spouse's passing and refused getting married, stating that her husband was the one and only love in her life.

She was reported to act oddly for a brief period of time soon after her spouse's death, blaming him for leaving her alone or trying to look attractive during the funeral; but they did not seek medical attention for these and she was reported to be "normal" until she had a hip joint replacement surgery 7 years ago. After the surgery, she became increasingly forgetful, started repeating the same sentences over and over, and lost her purse several times. There was a change in her character as well; always being a cheerful and outgoing person, she started to be less social. With these complaints, they saw a neurologist 5 years ago and a diagnosis of $\mathrm{AD}$ was made and she was started on donepezil $5 \mathrm{mg}$ initially which was increased to $10 \mathrm{mg}$ after a month. Two years ago, she developed anxiety symptoms and an increasing restlessness and visual hallucinations and a $20 \mathrm{mg}$ of citalopram was added to her drug regimen which helped with her anxiety symptoms but paranoid delusions associated visual and auditory hallucinations spiked up and that was when she was referred to our clinic for the first time.

At her first presentation two years ago, she was on $10 \mathrm{mg}$ of donepezil, $40 \mathrm{mg}$ of citalopram daily. She had severe memory deficits and visual and auditory hallucinations during the night time. She also had paranoid delusions. She closed her windows and curtains tightly because she supposed someone was chasing after her. She reportedly also started to show interest in clothes and underwear with flamboyant colors. The patient had three siblings, two of which were also diagnosed with $\mathrm{AD}$; however genetic testing was denied by the family. Neuropsychological assessment demonstrated severe memory impairment, moderate deficit in executive functions, visuospatial ability, attention, and concentration. She scored 13 on the Mini-Mental Status Examination (MMSE) and 2 on the Clinical Dementia Rating Scale (CDR). She was recommended to take memantine (with a dose to be gradually increased to $20 \mathrm{mg}$ within a month) and $25 \mathrm{mg}$ quetiapine at night time. After 15 days from her new medication, hallucinations and delusions were gone.

Her present visit was mainly due to these recent ISB. Her neuropsychological evaluation remained unchanged with a MMSE of 13 and a CDR of 2 .

Routine blood tests (complete blood count, electrolytes, renal and liver function tests and vitamin B12 and folate levels) were within normal limits. Her EEG demonstrated diffuse background slowing without epileptiform activity.

An MRI scan was ordered to rule out any superimposing organic pathologies such as a recently developed infarct or a space occupying lesion; 


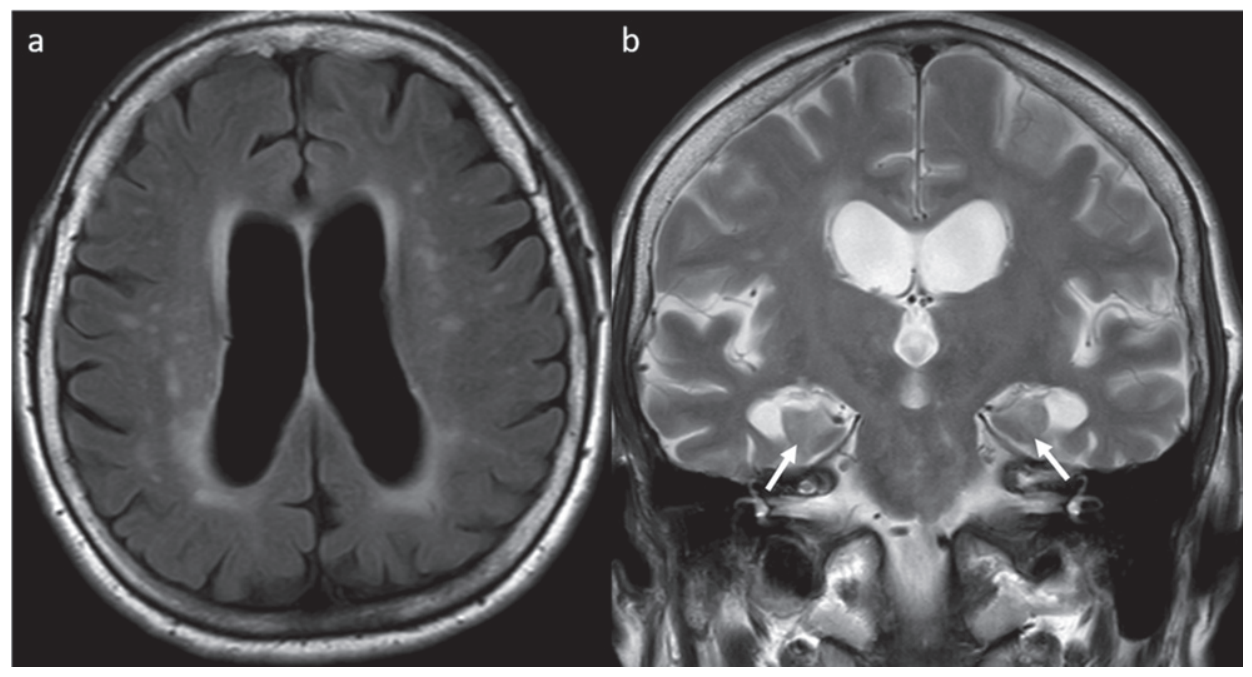

Fig. 1. MRI of the brain demonstrating mild-moderately enlarged lateral ventricles. a) Axial T2-weighted FLAIR demonstrating periventricular foci of prolonged T2 signal as well as scattered foci of T2 signal abnormalities compatible with small vessel ischemic disease. b) Coronal T2-weighted image demonstrating atrophy of the mesial temporal structures (arrows indicate diminished size of the hippocampi).

however it was unremarkable except signs of small vessel ischemic disease involving the periventricular white matter with scattered foci of $\mathrm{T} 2$ hyperintensities throughout the deep and subcortical white matter and moderate volume loss more prominently involving the mesial temporal structures (Fig. 1).

Her medication was re-evaluated and quetiapine $25 \mathrm{mg}$ that she had been on for 2 years, was stopped and she was prescribed aripiprazole with a dose of $2.5 \mathrm{mg}$ to be gradually increased to $10 \mathrm{mg}$ within a month. Phone interview about 2 weeks after the initiation of the aripiprazole treatment revealed that sexual remarks to the female staff members and other disinhibitory features subsided. She was less likely to get into physical contact with the female staff and other female residents of the nursing home, and her restlessness also improved. During the follow up visit after two months of the treatment, she was reported to be free of the symptoms without any ISB in the nursing home. She had increased personal interactions with other residents and the staff of both genders, without evolving into a disturbing point. No side effects were reported.

\section{DISCUSSION}

In elderly patients with dementia, cognitive deterioration, worsening in judgmental abilities, as well as personality changes, all contribute to changes in sexual attitude and behavior [2]. ISB may arise by several mechanisms. Neuropathologically, it may be a manifestation of the general disinhibition that results from damage to the lateral orbitofrontal circuit of the frontal/subcortical system [6]. Rarely dementia with ISB has been seen with bilateral amygdala lesions, as a symptom of the Kluver-Bucy syndrome (KBS). Kluver and Bucy, initially described this inappropriate sexual behavior as indiscriminate, with affected monkeys attempting to copulate with same-sex monkeys, other species, and even inanimate objects [7]. Our female patient showed hypersexual behavior solely to females but not to males. This suggests that her ISB should not be related to KBS.

There are published reports about hypersexuality among patients with dementia, head trauma, cerebral anoxia, multiple sclerosis, Parkinsonian disorders, and some other neurological disorders. Dementia, in particular, is a common cause of disordered sexual behavior, including hypersexuality. ISB is more common among patients with behavioral variant of frontotemporal dementia than in those with AD [8].

One of the unique features of the presented case is that, this is the first reported case in the literature of $\mathrm{AD}$ patient whose sexual orientation changed during the course of the disease. One possible hypothesis regarding this case, which might also be applied to similar cases of neurodegenerative diseases, is that she might have homosexual drives that could have been "in the closet" during her entire life and that might have become surfaced as the disease had progressed. However, because of the paucity of the 
publications in the literature we need more cases and hypotheses to draw conclusions.

There exist both nonpharmacological and pharmacological approaches for the treatment of ISB. It is usually accepted as appropriate to begin with nonpharmacological approaches in order to avoid unnecessary adverse effects [9]. Nonpharmacological treatment can be divided into environmental, behavioral (or cognitive-behavioral), and educational. These interventions should involve not only the patients but also families and the nursing staff for institutionalized subjects, bearing in mind that the aim is to try and promote an appropriate manifestation of sexual behavior [10]. If the nonpharmacological approach is unsuccessful at modifying ISB, pharmacological intervention may then be necessary. Case reports have described the use of a wide variety of medications in the male population, including antidepressants, gonadotropin releasing hormone analogues, antiandrogens, estrogens, anticonvulsants, antipsychotics, and antihistamines [9]. Our case showed no response to relatively high dose antidepressants. She also used a low dose antipsychotic (quetiapine) which showed no benefit for ISB.

There are two case reports of the use of quetiapine for treating ISB in the literature. One was an 85-year-old man with dementia, parkinsonism, and self-harming masturbation who showed no response to hormonal treatment and paroxetine. His symptoms disappeared after using quetiapine $25 \mathrm{mg}$, and did not recur in a follow-up period of 2 months, with no side effects or worsening parkinsonism reported $[2,11]$. Second case was a 61-year-old woman with Lewy body dementia, who showed a reduction of ISB after treatment with quetiapine which was titrated up to $75 \mathrm{mg}[2,12]$.

Two case reports described the successful use of haloperidol. A 70-year-old man who presented with excessive masturbation following a frontal lobe injury showed a marked reduction of symptoms with haloperidol $3 \mathrm{mg}$. [2, 13]. A 90-year-old man with $\mathrm{AD}$ and an array of behavioral symptoms including urethral masturbation with foreign bodies and sexual disinhibition had responded well to haloperidol $1.5 \mathrm{mg}$. [2, 14].

In the literature, there are two frontotemporal dementia (FTD) cases with ISB, treated successfully with aripiprazole. In one of cases, who was making frequent sexual remarks, sertraline and paroxetine were reported to be ineffective, but administering aripiprazole at $30 \mathrm{mg} /$ day for 2 weeks markedly improved the patient's condition [15]. The other FTD patient staying at an adult day-care center with ISB gradually worsening to a point which could not be handled any more. His ISB included exposing his genitals to other attendees and frequently making sexual remarks to the female staff. Upon his admission to the clinic, aripiprazole was initiated at $12 \mathrm{mg} /$ day, and the dose was gradually increased to $18 \mathrm{mg} /$ day in a week. Two weeks after the start of the medication, all of these symptoms subsided [16].

There is a report of FTD patient exhibiting pedophilia treated with combined therapy. A 60-yearold male was hospitalized after stalking, accosting, and attempting to molest children. After the clinical evaluation and investigations, he was diagnosed with FTD. The patient was started on paroxetine $20 \mathrm{mg}$, valproate $500 / 750 \mathrm{mg}$, and conjugated estrogens $0.625 \mathrm{mg}$. With this medical regimen and increased supervision, the patient had significant behavioral improvement, including a decrease of his sexual interest in children [17].

The second unique feature of the presented case is being the first reported case of ISB with AD treated with aripiprazole. Atypical antipsychotics are frequently used for the treatment of dementiarelated psychosis, despite FDA warnings because of increased mortality associated with the use of these medications in dementia patients [18].

ISB is one of the most difficult group of symptoms to manage in AD. ISB could hamper socialization which is very important for AD patients, as caregivers usually take it as embarrassing leading to the isolation of the patients and families.

In order to summarize, we believe this case is contributory to the literature for two reasons: 1) it is the first report of an AD patient presented with ISB of shifted sexual orientation; and 2) it is the first case of an AD with ISB treated successfully with aripiprazole.

\section{ACKNOWLEDGMENTS}

We would like to gratefully acknowledge Dr. Mario F. Mendez's opinions and contribution upon personal email communication.

\section{REFERENCES}

[1] Joller P, Gupta N, Seitz DP, Frank C, Gibson M, Gill SS (2004) Approach to inappropriate sexual behaviour in people with dementia. Can Fam Physician 59, 255-260.

[2] De Giorgi R, Series H (2016) Treatment of inappropriate sexual behavior in dementia. Curr Treat Options Neurol $\mathbf{1 8}, 41$. 
[3] Alagiakrishnan K1, Lim D, Brahim A, Wong A, Wood A, Senthilselvan A, Chimich WT, Kagan L (2005) Sexually inappropriate behaviour in demented elderly people. Postgrad Med J 81, 463-466.

[4] Levitsky AM, Owens NJ (1999) Pharmacologic treatment of hypersexuality and paraphilias in nursing home residents. $J$ Am Geriatr Soc 47, 231-234.

[5] Onishi J, Suzuki Y, Umegaki H, Endo H, Kawamura T, Imaizumi M, Iguchi A (2006) Behavioral, psychological and physical symptoms in group homes for older adults with dementia. Int Psychogeriatr 18, 75-86.

[6] Poetter CE, Stewart JT (2012) Treatment of indiscriminate, inappropriate sexual behavior in frontotemporal dementia with carbamazepine. J Clin Psychopharmacol 32, 137-138.

[7] Kluver H, Bucy PC (1937) Psychic blindness and other symptoms following bilateral temporal lobectomy in rhesus monkeys. Am J Physiol 119, 352-353.

[8] Mendez MF, Shapira JS (2013) Hypersexual behavior in frontotemporal dementia: A comparison with early-onset Alzheimer's disease. Arch Sex Behav 42, 501-509.

[9] Cross BS, DeYoung GR, Furmaga KM (2013) High-dose oral medroxyprogesterone for inappropriate hypersexuality in elderly men with dementia: A case series. Ann Pharmacother 47, e1.

[10] Ibrahim C, Reynaert C (2014) Hypersexuality in neurocognitive disorders in elderly people - a comprehensive review of the literature and case study. Psychiatr Danub 26(Suppl 1), 36-40.
[11] MacKnight C, Rojas-Fernandez C (2000) Quetiapine for sexually inappropriate behavior in dementia. J Am Geriatr Soc 48, 707.

[12] Prakash R, Pathak A, Munda S, Bagati D (2009) Quetiapine effective in treatment of inappropriate sexual behavior of Lewy body disease with predominant frontal lobe signs. Am J Alzheimer Dis Other Demen 24, 136-140.

[13] Kobayashi T (2004) Effect of haloperidol on a patient with hypersexuality following frontal lobe injury. Psychogeriatrics 4, 49-52.

[14] Rosenthal M, Berkman P, Shapira A, Gil I, Abramovitz J (2003) Urethral masturbation and sexual disinhibition in dementia: A case report. Isr J Psychiatry Relat Sci 40, 67-72.

[15] Reeves RR, Perry CL (2013) Aripiprazole for sexually inappropriate vocalizations in frontotemporal dementia. J Clin Psychopharmacol 33, 145-146.

[16] Nomoto H, Matsubara Y, Ichimaya Y, Arai H (2017) A case of frontotemporal dementia with sexual disinhibition controlled by aripiprazole. Psychogeriatrics 17, 509-510.

[17] Mendes MF, Chow T, Ringman J, Twichell G, Hinkin CH (2000) Pedophilia and temporal lobe disturbances. J Neuropsychiatry Clin Neurosci 12, 71-76.

[18] De Deyn PP, Drenth AF, Kremer BP, Oude Voshaar RC, Van Dam D (2013) Aripiprazole in the treatment of Alzheimer's disease. Expert Opin Pharmacother 14, 459-474. 